\title{
Milled versus moulded mock-ups based on the superimposition of 3D meshes from digital oral impressions: a comparative in vitro study in the aesthetic area
}

Francesca Cattoni ${ }^{1,2}$, Giulia Tetée ${ }^{2,3}$, Alessandro Mauro Calloni ${ }^{1,2}$, Fabio Manazza ${ }^{1,2}$, Giorgio Gastaldi ${ }^{1,4}$ and Paolo Capparè ${ }^{1,2^{*}}$ (D)

\begin{abstract}
Background: Aesthetic porcelain veneers proved to be a long-term reliable prosthetic solution, ensuring minimal invasiveness. The use of veneers requires an adhesive cementation technique, so maintaining as much enamel as possible is to ensure lasting success. A diagnostic mock-up is a key tool that allows a preview of the outcome of the aesthetic restoration: it is obtainable both in an analog and digital way. With the recent developments in impression technology and the ever so fast growing use of CAD-CAM technologies it is useful to understand the pros and cons of either one of these techniques (analog and digital) in order to identify the easier and more convenient workflow in aesthetic dentistry.
\end{abstract}

Methods: After taking pictures and impressions of the dental arcs of a patient in need of aesthetic rehabilitation, 52 resin models were produced and a digital drawing of the smile was outlined. Both an analog and a digital wax-up were obtained from two of the 52 models: the latter was obtained using digital impressions and a dedicated software. The analog wax-up was then used to produce 25 matrices that have later been used to mould 25 resin mock-ups using a traditional moulding protocol (Control Group - CG). The digital wax-up was used to mill 25 PMMA mock-ups. Each mock-up, both milled and moulded (total 50), was then laid on the other 50 resin models as a digital impression of it was taken. The STL. files of the milled mock-ups were compared with the 3D CAD wax-up made using a specific software. The STL. files of the analog printed mock-ups were compared with the traditional wax-up design. A statistical analysis was carried out to evaluate the difference between the groups.

Results: The statistical analysis showed a significant difference $(P>0.01)$ between the mean value of the distance between the points of the overlapping STL. meshes in GC $(0.0468 \mathrm{~mm})$ and in TG (Test Group - TG) $(0,0109 \mathrm{~mm})$.

Conclusions: The study showed a difference in accuracy between traditional moulded and milled mock-ups compared to their original wax-up. The data analysis reports that the digital method allows for greater accuracy. Within the limitations of this study, a fully digital workflow is to considered more reliable when it come to creating an esthetic mockup: the digital procedure has been shown to be more accurate than the one made manually which is much more operator dependent and it brings an increase to the chance of error, and that could ultimately affect the final result.

Keywords: Digital planning, Digital smile design, Mock up, Milling mock up, Digital workflow

\footnotetext{
* Correspondence: cappare.paolo@hsr.it

${ }^{1}$ Dental School, Vita-Salute San Raffaele University, Milan, Italy

2Department of Dentistry, IRCCS San Raffaele Hospital, Milan, Italy

Full list of author information is available at the end of the article
}

(c) The Author(s). 2019 Open Access This article is distributed under the terms of the Creative Commons Attribution 4.0 International License (http://creativecommons.org/licenses/by/4.0/), which permits unrestricted use, distribution, and reproduction in any medium, provided you give appropriate credit to the original author(s) and the source, provide a link to the Creative Commons license, and indicate if changes were made. The Creative Commons Public Domain Dedication waiver (http://creativecommons.org/publicdomain/zero/1.0/) applies to the data made available in this article, unless otherwise stated. 


\section{Background}

In recent years, the expectations of dental patients regarding aesthetic appearance have increased greatly. Aesthetic results have already reached comparable importance to masticatory function $[1,2]$. Porcelain veneers proved to be a long-term reliable solution, ensuring maximum aesthetics success and minimal invasiveness $[3,4]$. It is a priority to the clinician to pursue the least invasive procedure in every prosthetic restoration, preserving as much natural tooth structure as possible and respecting surrounding soft tissues [5]. Furthermore, since the use of veneers requires an adhesive cementation technique, maintaining as much enamel as possible is to ensure lasting success [6]. A diagnostic mock-up is to be intended as a tool that allows a better understanding of the patient's aesthetic expectations previewing the outcome of the aesthetic restoration, at a stage where it is still very easy to make changes according to patient's requests. It improves the communication with the patient, allowing prosthetic restorations to be achieved more successfully $([7,8])$. Moreover, a protocol that uses a diagnostic mock-up to guide the preparation has proved to be more conservative than a classical non-guided preparation made by the clinician [9]. As reported by Magne et Al., a veneer preparation driven by the final volume of the restoration (a diagnostic mock-up) allows for more enamel preservation, avoiding unnecessary over-preparation by only removing the structure needed to create proper prosthetic thicknesses, and more predictable outcome in terms of bonding, biomechanics and final aesthetics [10]. According to Coachman's protocol, the realization of the diagnostic wax-up is preceded and guided by the Digital Smile Design, which has proved to be a fundamental and useful tool for improving communication and patient's acceptance of the dental procedure [11-15]. This articulated workflow requires several steps that can lead to various inaccuracies. For instance, the mock-up molding phase on the existing tooth appears to be a very complex and heavily operator-dependent process. The most common problems related to the resin mock-ups are: the unevenly balanced positioning of the matrix, the inhomogeneous pressure during resin hardening, the difficulty in remove excess resin and the while finishing part to get a good final result [10]. Result that, if excessively discordant from what was promised and

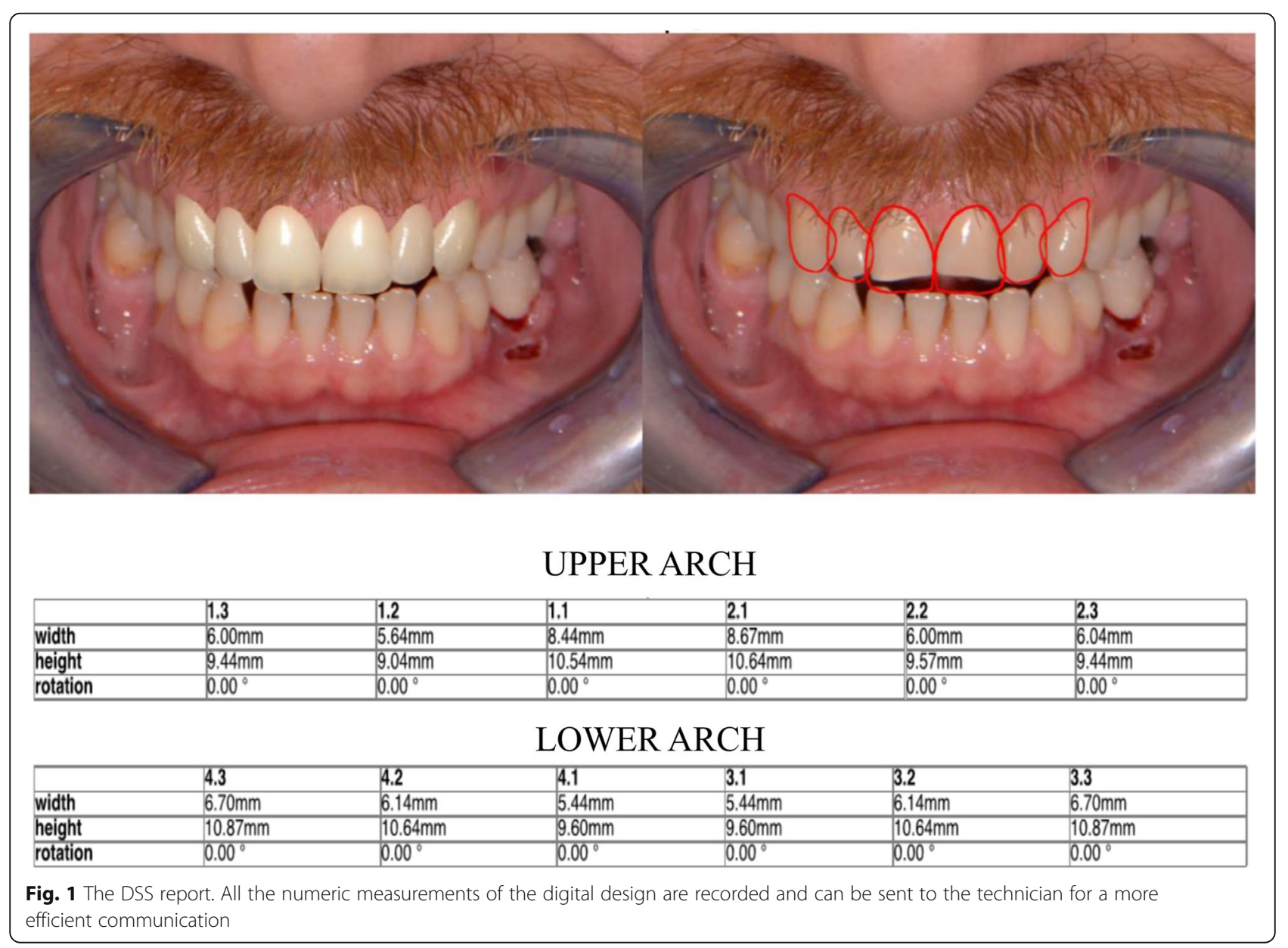


evaluated with the patient through the DSS software preview, could cause communication problems, misunderstanding and disappointment, or even the need to repeat the procedure, causing a waste of time and increasing the number of appointments required. Today, in attempt to minimize the chance of error and to shorten working times, the clinician can rely on effective smile planning tools and CAD / CAM systems (3D-Lynx Srl. Varese, Italy). Such systems, as shown by McLaren et Al., have proved their reliability in the realization of adhesive restorations in aesthetic areas [16]. A dedicated digital smile planning software with both two-dimensional and three dimensional features, is able to obtain excellent results in a simple, standardized and less operator-dependent way (3D-Lynx Srl. Varese, Italy). The aim of this study is to evaluate the traditional mock-up production method, which involves mock-up molding with a silicone matrix of the wax-up, compared to an exclusively digital workflow, which consists of mock-up milling from a CAD design, based on a digital optic impression. The accuracy of the two different types of mock-ups was compared each to their specific design and diagnostic wax-up.

\section{Methods}

A patient (male) in need of an additive restoration in the anterior area was selected in the dentistry department of IRCCS San Raffaele Hospital. Diagnostic pictures where taken during the first appointment, as well as analog polyether impressions of the upper arch (Impregum Penta, $3 \mathrm{M}$ ESPE, Sain Paul, Minn. USA). The virtual drawing of the final restoration was then obtained using a digital smile design software called DDS-2D (3D-Lynx Srl. Varese, Italy) (Fig 1). Starting from two photos of the patient, a extraoral shot (Fig. 2) with a maximum smile and an intra-oral one with slightly disclosed dental arches (Fig. 3) a digital drawing of the new smile was obtained and shown to the patient. This drawing was based

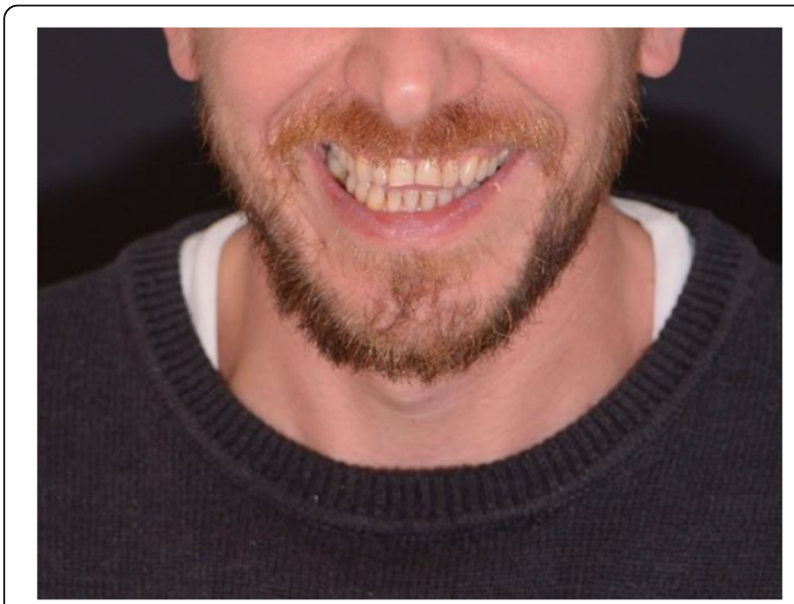

Fig. 2 Extra-oral photograph with with maximum smile

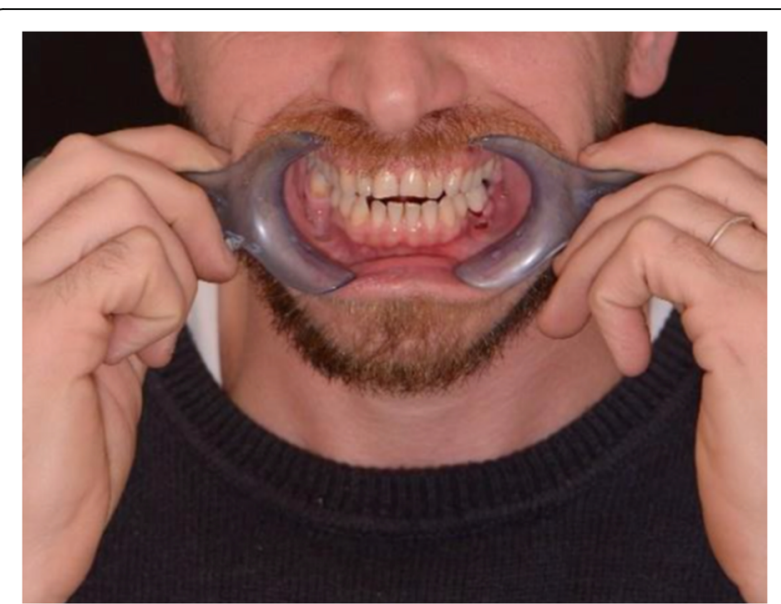

Fig. 3 Intraoral photograph with slightly spaced teeth, with specific glasses

on standard dental shapes included in a library inside the 2D software (Fig. 4). The digital restoration project was then realized (Fig. 5). This study was approved by the ethical board of the IRCCS San Raffaele Hospital of Milan (9/INT/2015). The patient provided their informed consent in writing.

\section{Traditional wax-up (control group)}

Fifty-two resin models were obtained from the analog impressions: one of them was used to produce a traditional wax-up by the technician, one was scanned using a laboratory desktop scanner (Scanner S-6000, Zirkonhzan Srl, Gais BZ) finally obtaining a STL file (Fig. 6) that allowed the design of a diagnostic mock-up using the Smile Design Software 3D-CAD expansion DSS-3D (3D-Lynx s.r.l., Varese, Italy). For the analog diagnostic wax-up, the software report provided the technician with all the images (Fig. 1) and all the operations performed by the clinician, indicating, with linear measures, how lengthened or shortened each measure had been during the design process. A intra-oral scanner (Dental Wings Intraoral Scanner, Dental Wings, Montreal, Canada) was then used to generate a STL file of the wax-up itself (Fig. 7).

Twenty-five silicone matrices (Fig. 8) were produced starting from the traditional wax-up (Fig. 9) (CG - Control group) and they were then used to mould a dual curing compost resin mock-up (Protempt 4, 3 M ESPE, Saint Paul, Minn. USA) on 25 of the remaining 50 resin models. The other 25 were used as a base for the 25 milled resin mock-ups to be laid upon (TG - Test Group). Those milled resin mock-ups were obtained by the STL - CAD project. Finally a digital impression of each of the 50 mock-ups (both moulded and milled) was taken with an integral scanner (Dental Wings Intraoral Scanner, Dental Wings, Montreal, Canada) and later analyzed. 


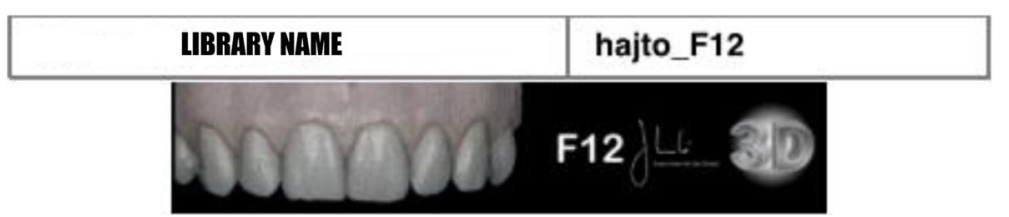

Fig. 4 The selected 3D teeth library

\section{Digital wax-up (test group)}

In order to obtain a digital wax-up, the STL. files of the patient's dental arches were uploaded to DSS 3D, a software which is the direct 3D implementation of the aforementioned DSS 2D. This software is able to align the patient's photograph to the digital model and support. The CAD software allowed us to design a threedimensional digital wax-up directly on the model. 25 resin mock-ups (PMMA - polymethil methacrylate) were then milled from the from the digitally planned 3D waxup. Each of these were then laid on the other 25 plaster models and as an optical digital impression was detected with the aforementioned intra-oral scanner. All the digital impressions of milled mock-ups, the moulded mock-ups, the wax-up and the STL. file of the CAD design were then uploaded to a lab software (OpenText Exceed 2017-EIM-Waterloo, ON,Canada). A superimposition and segmentation of the digital files have been performed to ensure that the impressions were cut out all the same way so the comparisons could not be affected by the different extensions of the original scans. Using a comparison software by (CloudCompare, https://www.cloudcompare.org) (Fig. 10), the STL files of the digital impressions, of the 25 manually moulded mock-ups were compared with the digital impressions of the traditional analog diagnostic wax-up while the STL files of the milled mock-ups were compared with the CAD designed project made with CAD - DSS 3D software (3D Lynx - Varese, Italy). The Stl. Files were segmented to make sure that only the area of the wax up was taken into consideration during the comparison. This was done by overlapping the two meshes with a

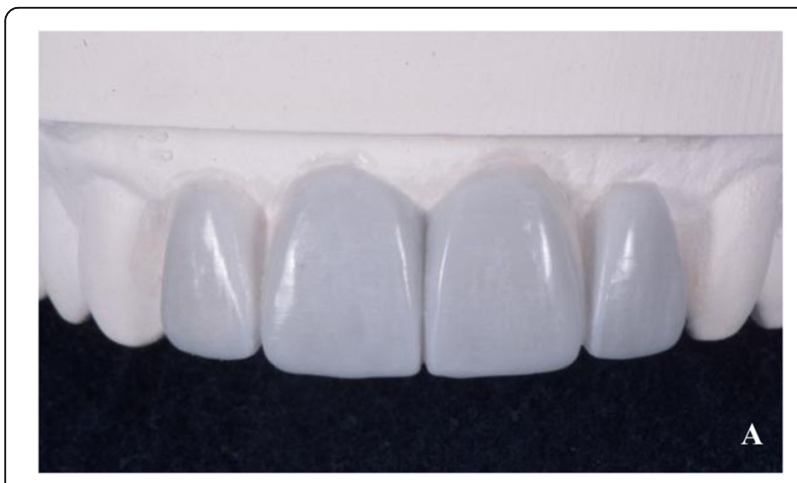

Fig. 5 Diagnostic mock-up "three point" manual alignment technique and measuring the average distance between the points of each one. This procedure represented also an index of the actual volumetric difference between them. Finally, the two methods were compared, evaluating which carried more errors and which remained more faithful to their design. Statistical analysis was carried out with SPSS-Student Ttest, which allows to compare the mean values of two non-coupled data sets.

\section{Results}

Results has shown specific areas of accumulation of errors and deviations: the cervical margin and the incisal edge (Fig. 10). The reasons why these particular areas exhibited major alterations are likely to be that the area of the cervical margin is the point where the excess of resin is removed from the silicone matrix, a procedure that is understandably difficult to replicate, while the differences found at the incisal edge are plausibly associated with variations in the pressure exerted by the operator on the silicone matrix to keep it in place during the hardening phase of the resin. The incisal edge, in particular, is of great aesthetic importance since it is a focal point for the observer; excessive variations in this area between the design and mock-ups can surely upset the patient, who is able to perceive the diversity of what has been promised with what he is really trying in his mouth.

The statistical analysis showed a significant difference between the two types of mock-ups. The null hypothesis that claims that the differences between the two groups were due to chance must be therefore rejected. The result obtained thus showed a clear difference in accuracy between moulded and milled mock-ups compared to their design. In the first case, not only moulded mockups diverged significantly from the diagnostic wax-up, demonstrating less accuracy (how much a measure is

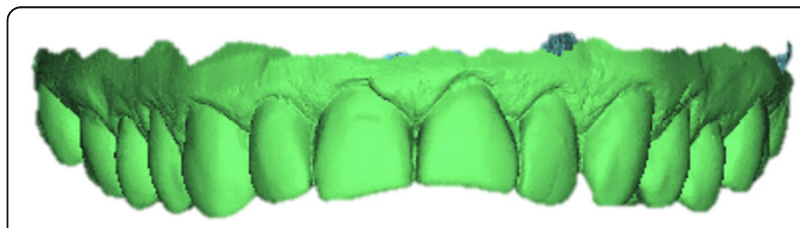

Fig. 6 STL file of the initial situation 


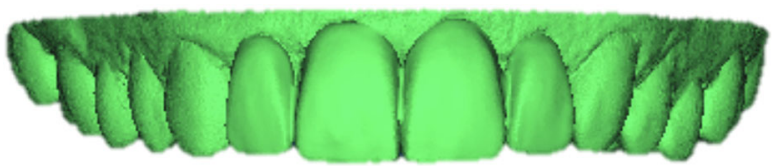

Fig. 7 CAD design of the digital wax-up

close to the true value of the size), but they also denoted a lesser degree of precision (how much measures are close to each other), indicated by the variance compared to the sample average (variance of moulded mock-ups: 0.0004). The milled mock-ups, on the contrary, were much more faithful to their CAD design, since the only error existing is that of the milling machine during the production phase, certainly negligible (variance of milled mock-ups: 0.00002) (Fig. 11). The milled mock-ups were therefore more accurate and precise. The use of the software allowed the comparison between the mean value of the distance between the points of the meshes superimposed on each other. Comparing the results obtained from the 50 evaluations performed, it was graphically very clear how the degree of overlap between moulded mock-ups and the diagnostic wax-up was significantly lower than that between milled mock-ups and the relative CAD design (Fig. 10).

From a more careful assessment, it was noted that the areas of accumulation of major deviations, which reflect errors during the realization, were consistently related to the portion of the cervical margin and the incisal edge.

The statistical analysis showed that the difference between mean value of the distance between the points of the meshes superimposed in the moulded mock-up group $(0.0468 \mathrm{~mm})$ compared to the milled mock-up group $(0.0109 \mathrm{~mm})$ was statistically significant $(P<0.01$; $P=0.300000000326)$ (Fig. 12).

\section{Discussion}

The aim of this study is to evaluate which method allows for the closest match with the initial wax up design and to find which stage of the production is affected by the



Fig. 8 The silicon index used to mould the mock-up



Fig. 9 One of the moulded mock-ups

greatest loss of precision, compromising the final result. In addition to the geometric and volumetric comparison between the different mock-ups and the respective waxups, the ease of execution for an unexperienced user has also been evaluated for each technique, since all the tests were carried out by an inexperienced operator. Many studies have shown how a mock up based approach can enable the clinician to provide patients with predictable aesthetics, since this particular kind of tool works with the psychology of patients, improving their attitude and compliance towards the treatment [10]. When starting a case, it is best for the practitioner to have in mind the end result since this has been shown to be vital in cases where the anterior teeth morphology is to be changed. A diagnostic wax-up can enhance the predictability of treatment by modeling the desired result in wax prior to treatment. It is critical to correlate the wax-up to the patient to avoid a result that appears optimal on the casts but does not correspond to the patient's smile [15-24]. Sancho-Puchades et al., point out that the use of a mock up will only be effective in an additive reconstructive case, while in subtractive cases it has to be used later in the treatment, after a minimum preparations of the natural teeth [25]. Wax ups and mock ups are reported to be extremely useful also for periodontal surgeons as tools used to perform crown lengthening procedures to enable future restorations in specific cases [13]. Results has shown specific areas of accumulation of errors and deviations; the cervical margin and the incisal edge. The reasons why these particular areas exhibited major alterations are likely to be that the area of the cervical margin is the point where the excess of resin is removed from the silicone matrix, a procedure that is understandably difficult to replicate, while the differences found at the incisal edge are plausibly associated with variations in the pressure exerted by the operator on the silicone matrix to keep it in place during the hardening phase of the resin. From the perspective of the impression techniques used in this study, the results showed that a 




Fig. 10 Graphical evaluation of printed and milled mock-up overlays for each of the 10 respective models in occlusal view, made with CloudCompare software

digital workflow was to be considered preferable in the hands of an unexperienced operator. Studies in literature claim that even though material such as poly-vinyl siloxane present great accuracy, digital impression techniques seem to be superior in terms of time and material saving; at the same time, said techniques lack in repeatability and this aspect represents a problem in need of solution [2]. As it was shown by the works of Gherlone et al. digital impression techniques manage to create an accurate physical model significantly improving efficiencies for the dental team and streamlining the workflow [17]. As far as the whole digital technique is concerned, a big role is played by the preview of the final result obtained through Digital Smile Design protocol. The use of 


\begin{tabular}{ccc}
\hline Number of samples & 25 & 25 \\
Mean & 0,046842916 & 0,010395569 \\
Variance & 0,000417003 & 0,000016484 \\
Standard Dev. & 0,020420642 & 0,004059990 \\
Student's T-Test & $\mathrm{P}=0,000000003256218$ \\
& \\
Difference between mean values was statistically significant per $\mathrm{P}>0,01$ \\
\hline
\end{tabular}

Fig. 11 Comparing mean values of two non-coupled data sets, with different variance

a smile designing software allows for an interdisciplinary collaboration between practitioners and this seems to improve the decision making process, ultimately decreasing the amount of intra-oral adjustments [12-28]. This tool allows the patient to preview the prosthetic result directly on a picture; it also provides the dental technician with all the necessary information on the execution of the work through a detailed report.

\section{Conclusions}

The study showed a difference in accuracy between traditional moulded and milled mock-ups compared to their original wax-up. The data analysis reports that the digital method allows for greater accuracy. Compared to the milled ones, the use of moulded mock ups would resolve in less accuracy of the mockup itself making it more difficult for the patient to visualize the final result

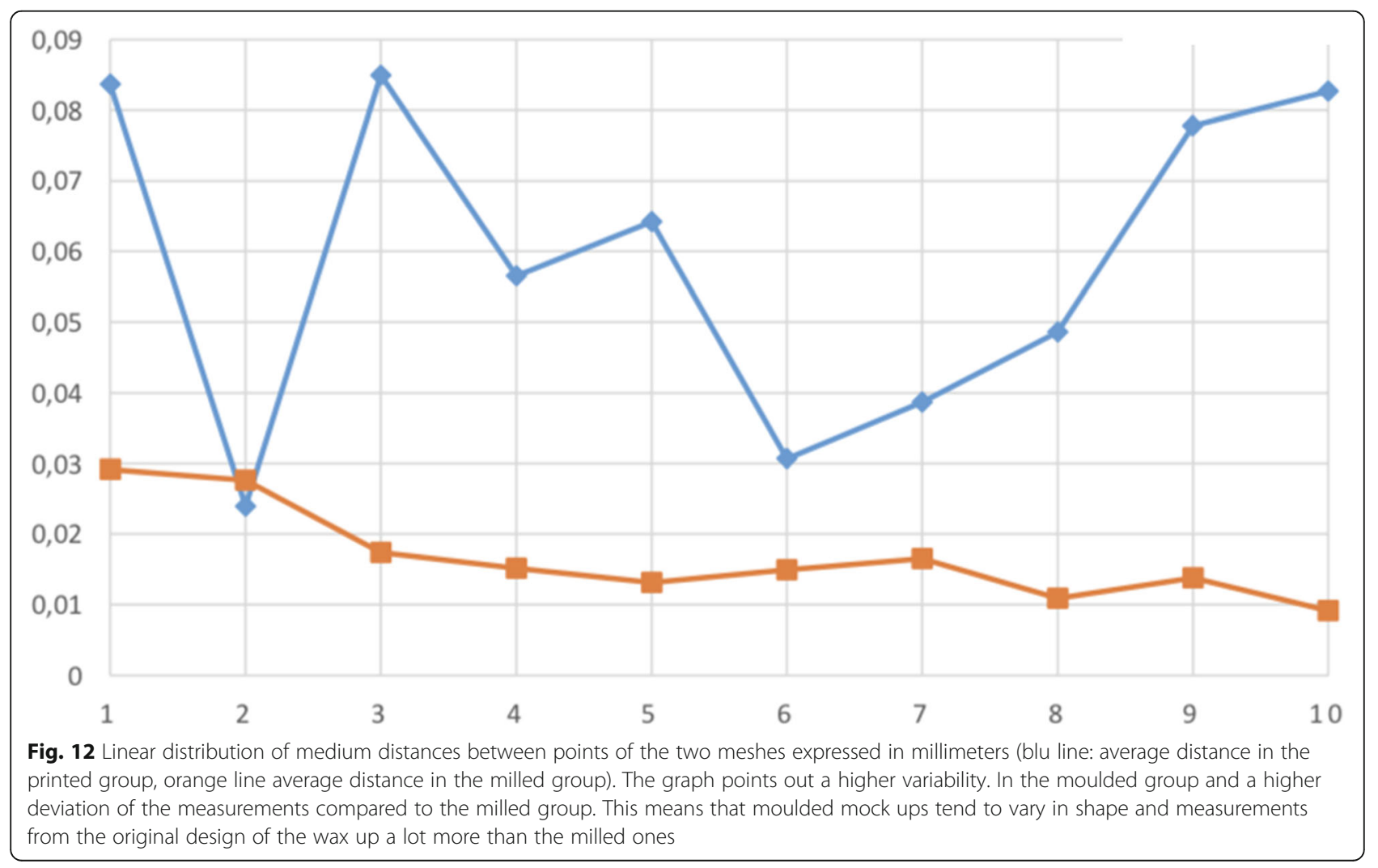


while wearing it therefore compromising the validity and acceptance of the entire prosthetics treatment plan. Within the limitations of this study, a fully digital workflow consisting in digital impression, digital wax up and milling technology is to considered more reliable when it comes to creating an esthetic mockup: the manual procedure has ben proven to be much more operator dependent and it brings an increase to the chance of error, and that could ultimately affect the final result.

\section{Abbreviations}

CG: Control Group; PMMA: Polymethil methacrylate; TG: Test Group

\section{Acknowledgements}

Not applicable.

\section{Authors' contributions}

AC and FM designed the study, FC and AC did the acquisition of data and analysis, FC and PC interpreted the data, PC and GT have drafted the work or substantively revised it. The last revison by GG. All Authors read and approved the final submitted version of the manuscript (and any substantially modified version that involves the author's contribution to the study);

\section{Funding}

This study has not any funder.

\section{Availability of data and materials}

All materials described in this manuscript including all relevant raw data, will be freely available to any scientist wishing to use them for non-commercial purposes, without breaching participant confidentiality. The data of this research is available from Paolo Capparè (corresponding author).

\section{Ethics approval and consent to participate}

This study was approved by the ethical board of the Scientific Hospitalization and Care Institutes San Raffaele Hospital of Milan (IRCSS) (9/INT/2015). All patients provided their informed consent in writing.

\section{Consent for publication}

All authors agree to publish the data collected and all authors read and approve the manuscript

Written informed consent for publication of potentially identifying

information was obtained from the subject of our manuscript.

\section{Competing interests}

The authors declare that they have no competing interests.

\section{Author details}

'Dental School, Vita-Salute San Raffaele University, Milan, Italy. ${ }^{2}$ Department of Dentistry, IRCCS San Raffaele Hospital, Milan, Italy. ${ }^{3}$ Specialisation School in Oral Surgery, Vita Salute San Raffaele University, Milan, Italy. ${ }^{4}$ Unit of Oral Maxillofacial Surgery, San Rocco Clinical Institute, Ome, Brescia, Italy.

Received: 26 February 2019 Accepted: 27 September 2019

Published online: 29 October 2019

\section{References}

1. Dawood A, Purkayastha S, Patel S, Mackillop F, Tanner S. Microtechnologies in implant and restorative dentistry: a stroll through a digital dental landscape. Proc Inst Mech Eng H. 2010;224(6):789-96.

2. Ting-Shu S, Jian S. Intraoral digital impression technique: a review. J Prosthodont. 2015:4(24):313-21.

3. Joda T, Zarone F, Ferrari M. The complete digital workflow in fixed prosthodontics: a systematic review. BMC Oral Health. 2017;17(1):124.

4. Peumans M, Van Meerbeek B, Lambrechts P, Vanherle G. Porcelain veneers: a review of the literature. J Dent. 2000;28(3):163-77.

5. Little D. The Impact of Aesthetics in Restorative Treatment Planning. Dent Today. 2015;34(5):104, 106-07.
6. Gurel G, Morimoto S, Calamita MA, Coachman C, Sesma N. Clinical performance of porcelain laminate veneers: outcomes of the aesthetic preevaluative temporary (APT) technique. Int J Periodontics Restorative Dent. 2012;32(6):625-35.

7. Granell-Ruiz M, Fons-Font A, Labaig-Rueda C, Martinez-Gonzalez A, RomanRodriquez JL, Solà-Ruiz MF. A clinical longitudinal study 323 porcelain laminate veneers. Period study from 3 to 11 years. Med Oral Patol Oral Cir Bucal. 2010;15(3):531-7.

8. Gurel G. Porcelain laminate veneers: minimal tooth preparation by design. Dent Clin N Am. 2007;51(2):419-31.

9. Buonocore MG. A simple method of increasing the adhesion of acrylic filling materials to enamel surfaces. J Dent Res. 1955;34(6):849-53.

10. Reshad M, Cascione D, Magne P. Diagnostic mock-ups as an objective tool for predictamble outcomes with porcelain laminate veneers in esthetically demanding patients: a clinical report. J Prosthet Dent. 2008;99(5):333-9.

11. Santos DMD, Moreno A, Vechiato-Filho AJ, Bonatto LR, Pesquiera AA, Junior MCL, de Medeiros RA, da Silva EV, Goiato MC. The importance of the lifelike esthetic appearance of all-ceramic restorations on anterior teeth. Case Rep Dent. 2015. https://doi.org/10.1155/704348.

12. Veneziani M. Ceramic laminate veneers: clinical procedures with a multidisciplinary approach. Int J Esthet Dent. 2017;12(4):426-48.

13. Gurrea J, Bruguera A. Wax-up and mock-up. A guide for anterior periodontal and restorative treatments. Int J Esthet Dent. 2014;9(2):146-62.

14. Magne P, Belser UC. Novel porcelain laminate preparation approach driven by a diagnostic mock-up. J Esthet Restor Dent. 2004;16(1):7-16.

15. Simon H, Magne P. Clinically based diagnostic wax-up for optimal esthetics: the dagnostic mock-up. J Calif Dent Assoc. 2008;36(5):355-62.

16. Coachman C, Calamita MA, Sesma N. Dynamic documentation of the smile and the 2D/3D digital smile design process. Int J Periodontics Restorative Dent. 2017;37(2):183-93.

17. Gherlone EF, Ferrini F, Crespi R, Gastaldi G, Capparé P. Digital impressions for fabrication of definitive all-on-four restorations. Implant Dent. 2015:24(1):125-9.

18. Gherlone EF, Capparé P, Vinci R, Ferrini F, Gastaldi G, Crespi R. Conventional versus digital impressions for all-on-four restorations. Int J Oral Maxillofac Implants. 2016;31(2):324-30.

19. Schmitter M, Seydler B. Minimally invasive lithium disilicate ceramic veneers fabricated using chairside CAD/CAM: a clinical report. J Prostet Dent. 2012; 107(2):71-4.

20. Gherlone EF, Mandelli F, Capparé P, Pantaleo G, Traini T, Ferrini F. A 3 years retrospective study of survival for zirconia-based single crowns fabricated from intraoral digital impressions. J Dent. 2014;42(9):1151-5.

21. Cattoni F, Mastrangelo F, Gherlone EF, Gastaldi G. A New Total Digital Smile Planning Technique (3D-DSP) to Fabricate CAD-CAM Mockups for Esthetic Crowns and Veneers. Int J Dent. 2016. https://doi.org/10.1155/6282587.

22. Seydler B, Schmitter M. Esthetic restoration of maxillary incisors using CAD/ CAM chairside technology: a case report. Quintessence Int. 2011;42(7):533-7.

23. Ercus S, Chung E, McLaren E. Esthetics with minimal tooth preparation achieved through a digital approach. Compend Contin Educ Dent. 2013; 34(6):428-31.

24. Lin WS, Zandinejad A, Metz MJ, Harris BT, Morton D. Predictable restorative work flow for computer-aided design/computer-aided manufacturefabricated ceramic veneers utilizing a virtual smile design principle. Oper Dent. 2015:40(4):357-63.

25. Sancho-Puchades M, Fehmer V, Hämmerle C, Sailer I. Advanced smile diagnostics using CAD/CAM mock-ups. Int J Esthet Dent. 2015;10:374-91.

26. Fasbinder DJ. Computerized technology for restorative dentistry. Am J Dent. 2013;26(3):115-20

27. Murchison DF, Burke FJ, Worthington RB. Incisal edge reattachment: indications for use and clinical technique. Br Dent J. 1999;186(12):614-9

28. Coachman C, Paravina RD. Digitally enhanced esthetic dentistry - from treatment planning to quality control. J Esthet Restor Dent. 2016;28(Suppl 1):S3-4

\section{Publisher's Note}

Springer Nature remains neutral with regard to jurisdictional claims in published maps and institutional affiliations. 